\title{
MECANISMOS DE ATENUACIÓN ENTRE EL DECIR Y LO DICHO: PROCESOS DE ENUNCIACIÓN A TRAVÉS DE LA PARTÍCULA DISCURSIVA DIGAMOS
}

\author{
MITIGATION DEVICES BETWEEN \\ TO SAY AND WHAT HAS BEEN SAID: ENUNCIATION PROCESSES \\ WITH THE DISCOURSE PARTICLE DIGAMOS
}

\author{
Shima SAlameh JimÉnez \\ Universitat de València / Universität Heidelberg \\ Grupo Val.Es.Co. / Grupo DPKog \\ Shima.Salameh@uv.es
}

Recibido: 20/12/2016

Aceptado: 30/05/2017

\begin{abstract}
Resumen
Este trabajo presenta los resultados obtenidos en el análisis de la partícula discursiva digamos en corpus coloquiales (Val.Es.Co. 2002 y 2.0.), aplicando el sistema de unidades, niveles y posiciones Val.Es.Co. (Briz y grupo Val.Es.Co. 2003, 2014) y la ficha Es. Por. Atenuación (Albelda et alii 2014). El resultado integra dos perspectivas de análisis para determinar si factores como la unidad ocupada o posición discursiva condicionan su polifuncionalidad.
\end{abstract}

PALABRAS CLAVE: Modelo Val.Es.Co., digamos, marcadores discursivos, atenuación.

\begin{abstract}
This study presents the results obtained from the analysis of the Spanish discourse particle digamos in colloquial corpus. The Val. Es.Co. proposal (Briz et alii 2003, 2014) and the Es.Var.Atenuación analysis sheet (Albelda et alii 2014) are employed in the analysis. The result integrates two perspectives of analysis through which it is possible to determine whether the unit occupied by the particle or the discourse position are conditions for the polyfunctionality of digamos.

KEY WORDS: VAM units model, digamos, discourse markers, mitigation.
\end{abstract}

Para citar este artículo / To cite this article: Salameh Jiménez, Shima (2018): Mecanismos de atenuación entre el decir y lo dicho: procesos de enunciación a través de la partícula discursiva digamos. García Ramón, Amparo y Soler Bonafont, María Amparo (Eds.): ELUA: Estudios de atenuación en el discurso, Anexo IV, págs. 85-100.

Enlace / Link: http://dx.doi.org/10.14198/ELUA2018.Anexo4.05 


\section{INTRODUCCIÓN}

En el ámbito del español ${ }^{1}$, las partículas y estructuras discursivas empleadas en la actividad del decir se han estudiado en diferentes trabajos de corte sincrónico (Fernández Bernárdez 2002; Schneider 2007; Grande Alija 2010, así como el Diccionario de Partículas Discursivas del Español (DPDE) (Briz, Pons y Portolés, en línea), el Diccionario de Partículas (DPD) (Santos Río 2003) y el Diccionario de Conectores y Operadores del Español (DCOE) (Fuentes 2010)). Una de las partículas discursivas con decir más frecuentes es digamos. Presenta mayor movilidad posicional que otras y, por tanto, mayor variación de ámbito: puede aparecer en posición inicial, medial o final, y su ámbito se amplía o reduce según funcione a nivel sintáctico, semántico o pragmático. Sin embargo, su polifuncionalidad (ejemplificación, reformulación, digresión, difusión del significado, atenuación pragmática o apoyo a la formulación), descrita en la bibliografía, presenta una caracterización compleja: debido a los procesos de cambio $^{2}$ que ha experimentado hasta convertirse en PD, digamos manifiesta rasgos explícitos de su origen verbal en numerosos contextos de uso ${ }^{3}$, así como valores pragmáticos, además de usos asociados a funciones propias de la vaguedad semántica (Zhang 1998).

Con el fin de sistematizar el tratamiento de digamos, este trabajo ofrece, a modo de primera aproximación, los resultados obtenidos en un análisis de muestras de digamos en conversaciones coloquiales. Para ello, se han utilizado los corpus de conversaciones coloquiales del grupo Val.Es.Co. (Briz y Grupo Val.Es.Co. 2002; Cabedo y Pons en línea) y se han aplicado dos herramientas de análisis: por un lado, el sistema de unidades, niveles y posiciones del grupo Val.Es.Co. (Briz y grupo Val.Es.Co. 2003; Grupo Val.Es.Co. 2014); por otro lado, la ficha metodológica para el análisis de la atenuación Es.Por.Atenuación (Albelda, Briz, Cestero, Kotwica y Villalba 2014).

El trabajo se organiza en los siguientes apartados: en el marco teórico ( $§ 2)$, por un lado, se abordarán cuestiones generales sobre atenuación ( $§$ 2.1.); por otro lado, se delimitarán los rasgos característicos de digamos (§2.2). Seguidamente (§ 3), se sintetizarán las características básicas del modelo de unidades, niveles y posiciones del grupo Val.Es.Co. (§ 3.1.) y las de la ficha de análisis de Es.Var.Atenuación (§ 3.2.). Se ofrecerán los resultados del análisis $(\S 4)$, obtenidos tras la extracción y estudio de las muestras de digamos en los corpus mencionados.

\section{MARCO TEÓRICO}

\subsection{Atenuación y cortesía social: partículas discursivas como mecanismo de atenuación}

Atenuación y cortesía lingüística son dos campos de investigación interrelacionados dentro del ámbito de la Pragmática, y que suelen darse simultáneamente. Sin embargo,

1 También a nivel internacional, estos elementos parentéticos han sido objeto de estudio en numerosos trabajos, entre otros: Thompson, S. \& A. Mulac (1991), Brinton, L. (2005); Schneider, S. (2007).

2 Principios como la estratificación o la persistencia (Hopper, 1996) son los que explican que, a pesar del desarrollo y fijación de nuevos valores en elementos que se gramaticalizan, se mantengan en cierto grado los significados de base (Hopper, P. 1996: 230-231). En español, partículas discursivas como mira, oye, ¿ves?, digamos, etc., manifiestan de forma muy clara estos principios, ya que el resultado de su gramaticalización es un producto discursivo con procedencia en una palabra léxica.

3 Salameh Jiménez (2015). 
como apuntan Albelda y Briz (2010: 238) es importante no confundirlos ${ }^{4}$ : mientras la cortesía, dentro del plano de la comunicación, es un "hecho social" que se extiende a distintas culturas, y para la que se pueden utilizar diferentes estrategias discursivas, la atenuación "es un hecho lingüístico", una "categoría pragmática al servicio de la comunicación" (Albelda y Cestero 2011) que puede emplearse, o no, con fines corteses. El uso de la atenuación permite modificar el impacto que generan muchos actos de habla (Fraser 1980: 342) y, consecuentemente, disminuir la afección de la imagen social de los interlocutores (FTA, Brown y Levinson $1987^{5}$ ).

Existen diferentes procesos de atenuación, según las condiciones comunicativas en las que se lleven a cabo o los recursos empleados para lograrla: ensalzamiento de la imagen del interlocutor, autoprotección de la imagen del hablante, protección de la imagen del interlocutor, o protección de ambas imágenes a la vez. Los siguientes ejemplos (1-2) son una muestra de ello:

(1) A: [tienes que] captarles la atención $\downarrow$ y como cada niño $\uparrow / /$ tienes que darle un poco a cada niño loo- lo suyo $\downarrow$ entonces $\uparrow / /$ y ¡claro! es muy difícil hoy en día eso $\downarrow / /$ quee los nanos $\uparrow$ yo creo que están desmadrados totalmente/ entre televisión $\uparrow /$ no sé cuántos $\downarrow /$ son- [ison MUY BRUTOS!/ yo los veo muy brutos]

B:

[sí $\downarrow$ es lo

que ocurre// lo que ocurre $\downarrow] /$ yo creo que quizás $\uparrow /$ están demasiadoo (CHASQUIDO) ajenos a la realidad
A: ¿sí?§
B: §aparte de que [la infancia=]
A: $\quad$ [aparte de]
B: =es de por sí $\uparrow$ pues eso $\downarrow$ [estar ajeno=]

(Corpus Valesco 2.0, Conversación 1, Intervenciones 174-182)

(2) B: §yo creo que te lo he demostrado/ que estoy bastante segura $\S$

A:

$\S$ mira $\downarrow$ yo te quiero... y cre- y creo que lo SABES/// pero NO/ no puedo DEMOSTRÁRTELO $\downarrow$ o sea no- no puedo dedicarte todo lo que tú necesitas

B: pero si yo no te pido que me lo demuestres

A: pero

(Corpus Val.Es.Co., ML.84.A.1: 75)

En (1), el hablante A manifiesta, con vehemencia, su opinión acerca de la educación que reciben los niños hoy en día: argumentos como la dificultad para captar la atención de los niños en clase, estar desmadrados o el exceso de televisión le llevan a defender la conclusión son muy brutos. El hablante B responde que, realmente, esto puede deberse a que los niños se encuentran ajenos a la realidad, y que la infancia conlleva que el niño se encuentre, normalmente, en ese estado. Al tratarse de una opinión opuesta a la del hablante A, B reduce

4 Los hablantes pueden desarrollar procesos de cortesía social no solo mediante estrategias de atenuación, sino también con mecanismos de intensificación (Albelda 2004).

5 Debido a que el objetivo del presente trabajo es abordar mecanismos de atenuación lingüística y, concretamente, aquellos vinculados con la marcación del discurso y la estructura conversacional, no se tratarán nociones como FTA, cortesía positiva, cortesía negativa, máximas de cortesía, etc. Para ello, consúltense referencias como Brown y Levinson (1987) o Lakoff (1989). 
la fuerza de su declaración con el fin de proteger su imagen: verbos en primera persona, como creo, le permiten explicitar la autoría de su intervención y atribuirse el contenido de lo dicho; adverbios como quizás evocan contextos de posibilidad, y no de absoluta certeza, con respecto a lo que se dice.

En el ejemplo (2), el hablante B realiza un acto de habla de reproche a A. Con marcas interactivas como ¿no? o tío, y difusores del significado como o algo o yo qué sé, B consigue reducir parcialmente su acto de habla, a pesar de que la actitud de su interlocutor le parezca inadecuada. Finalmente, la valoración hacia el comportamiento de A (me parece un poco $\mathrm{mal}$ ) está introducida mediante el cuantificador un poco, modificador externo que reduce el ataque a la imagen de $\mathrm{B}$, que en esta ocasión se vería afectada.

A los parámetros comunicativos propios del análisis pragmático, se suman otros elementos lingüísticos que contribuyen al proceso de atenuación: todas las lenguas, como vehículos de expresión de diferentes culturas (con coincidencias y divergencias en muchos puntos ${ }^{6}$ ), cuentan con una amplia variedad de mecanismos (verbales y no verbales) para atenuar (Blum-Kulka y Olshtain 1984; Brown y Levinson 1987; Haverkate 1994; Briz y Bravo 2004; Caffi 2007, entre otros), orientados a mitigar el contenido proposicional (atenuación semántico-pragmática) o la fuerza ilocutiva (atenuación pragmática), pero siempre bajo condiciones contextuales (Briz 1998).

Junto a los mecanismos de atenuación verbales (de tipo morfológico, sintáctico, semántico y pragmático-discursivo), combinados normalmente con rasgos prosódicos (Culpeper 2011), destaca el campo de las partículas discursivas ${ }^{7}$. Cuando ciertas partículas funcionan en contextos en los que el hablante pretende reducir la fuerza de sus actos de habla, el impacto en la imagen de su interlocutor, o reparar aquello que ya ha dicho, estas acaban asimilando este funcionamiento atenuador como propio de su significado de base. A su vez, los límites entre enunciado y enunciación se tornan borrosos: el hablante hace uso de las partículas para modificar lo que está diciendo, pero estas también quedan plasmadas en su discurso (lo dicho) mientras se construye.

Por esta razón, describir y sistematizar los rasgos de estas partículas (sobre todo a nivel estructural -sintáctico y/o posicional-) puede convertirse en una tarea abstracta si no se hace con las herramientas de análisis adecuadas.

\subsection{Digamos en español peninsular: propiedades básicas}

El español cuenta con diferentes partículas y construcciones discursivas con decir: pueden tener como base una única forma (digo, dicen, digamos), o bien combinarse con otros elementos (es decir, digo yo, ya te digo, no digamos, que digamos, digámoslo así, por así

$6 \quad$ A pesar del carácter universal de la noción de Face Threatening Act (FTA, Goffman 1955; Brown \& Levinson 1987), es sabido que las diferencias culturales tienen un reflejo directo en los mecanismos para desarrollar procesos de atenuación e intensificación en el marco de la cortesía social, e incluso en los modos de hacerlo -focalizar, por ejemplo, en los FTA positivos o negativos, ensalzar imágenes sociales o reducir la fuerza de los ataques-. Las culturas americana y china son muestra de ello (Wang 2011: 52): los resultados no serán los mismos si se produce un ataque a la imagen del interlocutor en una lengua o en otra, debido al poder determinante que los parámetros sociales poseen para ambas culturas.

7 El campo de las partículas discursivas ha sido altamente productivo desde su aparición a finales de la década de 1980 (Schourup 1985; Bazzanella 1986; Schiffrin 1987; Blakemore 1987; Fraser 1990, 1999; Fuentes Rodríguez 1989; Pons Bordería 1994, 1998; Briz y otros 2003; Loureda y Acín 2010; entre otros muchos). 
decirlo, etc.). Todas poseen valores orientados a la formulación discursiva o a la modalidad. Son verbos gramaticalizados, de uso frecuente en el ámbito oral coloquial, y van más allá de una lectura realizativa (González Ruiz 2006: 111), puesto que su interpretación no equivale al anuncio de la ejecución de una acción determinada mediante el habla, sino a marcar que el proceso de formulación discursiva se está desarrollando.

En el caso de digamos, varias propiedades permiten atribuirle la categoría de partícula discursiva y distinguirlo funcionalmente de las otras partículas mencionadas:

- Morfológicamente, digamos presenta propiedades óptimas para su funcionamiento en contextos de atenuación pragmática y vaguedad semántica: procede de un verbo en primera persona del plural, de presente de subjuntivo.

- Sintácticamente, digamos suele aparecer entre comas en textos escritos, o entre pausas en conversaciones coloquiales. No está, por tanto, integrado en la estructura sintáctica general y su análisis se sitúa en el nivel supraoracional, característica prototípica de las partículas discursivas. Este rasgo la convierte en un elemento sintácticamente independiente, razón por la que digamos presenta una movilidad estructural muy alta: puede aparecer en posición inicial, medial, e incluso final, pero con un ámbito muy variable.

- Semánticamente, digamos tampoco modifica el contenido proposicional del segmento en que se inserta: su supresión no daría lugar a una formulación agramatical o incompleta, sino a una pérdida de valores pragmáticos que van de la aproximación a la atenuación. Actúa, como suelen hacer las partículas discursivas, en el nivel del modus (intención comunicativa) y no del dictum (lo dicho), como sí haría un verbo decir integrado y sin gramaticalizar.

- Pragmáticamente, digamos permite al hablante explicitar un tipo de decir marcado en su contexto interactivo. Esto es necesario cuando se debe explicitar, de algún modo, que el sentido de su enunciación supera los límites de lo dicho en sentido literal.

Este último rasgo pragmático es determinante para digamos, que se aleja así del funcionamiento reformulador del también frecuente digo, del evidencial digo yo, o de las formas no digamos y que digamos (Grande Alija 2010), propias de la intensificación discursiva. Digamos permite atenuar, a nivel semántico-pragmático, en tres grados distintos: no-literalidad, aproximación o vaguedad semántica (3), atenuación pragmática (4), y apoyo formulativo $(5,6)$. Los siguientes ejemplos muestran las propiedades y grados mencionados:

(3) Es, por ejemplo, estudiando la organización columnar del córtex o el condicionamiento configural, como más ostensibles pueden hacerse las limitaciones de una psicología de la Forma tópicamente entendida. Es, digamos, enfrentándonos con las teorías evolutivas de Piaget como mejor cabe poner de manifiesto la insuficiencia del asociacionismo para dar cuenta de la estructura del pensamiento.

(José Luis Pinillos, Principios de Psicología, 1975)

En el ejemplo (3), el hablante no desea hablar de un enfrentamiento literal contra una teoría evolutiva, sino de una oposición de ideas consolidadas en el ámbito de la psicología. Por eso se trata de un valor no-literal, semánticamente borroso, que el DRAE trata como 
casos figurados o aproximativos: digamos "presenta la palabra o palabras que se dan como expresión aproximada de lo que se pretende significar". Asimismo, el DPDE repara en esta "no literalidad" de las expresiones sobre las cuales actúa el marcador.

(4) - La cuestión es que no funcionaba la comisión técnica...

-Para mí todo tiene que tener una función positiva. Digamos que había cosas con las que no estaba de acuerdo, pero no quiero comentar nada más sobre esto.

(La Vanguardia, 16/10/1995, Deportes)

En el ejemplo (4), se hace referencia a la función mitigadora de digamos, empleado a menudo "con el fin de atenuar lo dicho y evitar responsabilidades ante el interlocutor" (Fernández Bernárdez, en línea). Su uso no implica únicamente una modificación en la base semántica del segmento sobre el que digamos tiene ámbito, sino que reduce la fuerza ilocutiva del acto de habla afectado (en este caso, una aseveración). De este modo se evita, a nivel social, que la imagen del interlocutor y la del propio emisor puedan verse afectadas.

(5) -Para mí la revolución es siempre un proceso de transformación, siempre lo he concebido así, no asociándola, digamos, a acontecimientos más o menos folcróricos y a formas concretas de hacer esa revolución.

(Prensa, España, Cambio 16, no 987, 22/10/1990: Antonio Gutiérrez)

(6) Era era era, digamos, era un hombre un hombre bisagra, posiblemente No, no, era sencillamente en en en una especie de bipartidismo histórico.

(Oral, España, Magazines, 08/11/1983)

Por último, cuando el hablante no encuentra, en los casos de formulación inmediata, recursos lingüísticos adecuados para expresarse con claridad, puede emplear marcas que expliciten que su intervención está en desarrollo. De este modo, el hablante consigue un margen de tiempo para elaborar su mensaje y evitar una pérdida del turno conversacional. Los ejemplos (5) y (6) son muestras de este uso formulativo, muy frecuente en el registro coloquial. Mientras en (5) digamos permite al hablante continuar elaborando su mensaje (de ahí su posición puramente medial), (6) refleja el grado más extremo de apoyo formulativo en una intervención, junto con repeticiones, vacilaciones y rasgos propios de una enunciación dificultosa.

\section{HERRAMIENTAS DE ANÁLISIS}

\subsection{El sistema de unidades, niveles y posiciones del grupo Val.Es.Co. (Briz y grupo Val.Es.Co. 2003, 2014; Pons 2016)}

El modelo de segmentación en unidades, niveles y posiciones Val.Es.Co. (Briz y Grupo Val.Es.Co. 2003; Grupo Val.Es.Co. 2014; Pons 2016) es uno de los varios modelos de segmentación activos actualmente en el ámbito de la Pragmática, surgidos de la necesidad de analizar discursos conversacionales y textuales con herramientas distintas a las que brinda la sintaxis tradicional (Narbona 1989). 
El estudio pormenorizado del discurso, en su vertiente textual o conversacional, era el principal objtivo de campos como el Análisis del Discurso (AD), el Análisis de la Conversación (AC), o la Lingüística del Texto (LT), consolidados entre las décadas 1960-1970 como focos de interés en lingüística (Sacks, Jefferson y Schegloff 1974; Van Dijk 1979). Textos, conversaciones o entrevistas recogen ciertos fenómenos (incisos, solapamientos, escisiones conversacionales, vacilaciones, reinicios, etc.) que superan los límites oracionales y que requieren un tratamiento distinto, con otras unidades que expliquen y organicen su funcionamiento.

Escuelas como la de Birmingham (Sinclair 1975), el Grupo de la Sorbona (Morel y Rilland 1992) o el Grupo de Ginebra (Roulet et alii 1980, 2001; Roulet 1991) continuaron en esta línea de investigación, y sus trabajos son antecedentes teóricos directos del modelo de segmentación Val.Es.Co.

Este estudio aplica el modelo de unidades Val.Es.Co. porque sus características permiten un tratamiento descriptivo y sistemático de la partícula digamos:

- En primer lugar, la distinción entre unidades monológicas (las que se refieren al producto discursivo de un hablante) y dialógicas (las que plasman las relaciones interactivas entre los hablantes) lo convierte en una herramienta explicativa de la interacción, "ya sea esta más o menos dialogal, que no se encuentra en otros modelos" (Grupo Val. Es. Co. 2014: 15).

- En segundo lugar, el modelo distingue tres órdenes que aportan mayor precisión a la descripción de la partícula: estructural-interno, social-externo e informativo.

Siguiendo su propuesta más actualizada (2014), ocho son las unidades del modelo Val. Es.Co. (discurso, diálogo, intercambio / alternancia de turnos, intervención / turno, acto y subacto), tres las dimensiones de análisis (social, informativa y estructural), y cuatro las posiciones ligadas a cada unidad (inicial, medial, final e independiente) (Tabla 1):

\begin{tabular}{|l|l|l|l|}
\hline Nivel & \multicolumn{2}{|l|}{ Dimensiones } & \multirow{2}{*}{ Informativa } \\
& Estructural & Social & \\
\cline { 2 - 3 } & $\begin{array}{l}\text { Discurso } \\
\text { Diálogo } \\
\text { Intercambio }\end{array}$ & Alternancia de turnos & \\
\hline Monológico & $\begin{array}{l}\text { Intervención } \\
\text { Acto }\end{array}$ & Turno & \\
& & Subacto \\
\hline
\end{tabular}

Tabla 1. Unidades del sistema Val. Es. Co. (Grupo Val.Es.Co. 2014: 15).

Las unidades y posiciones seleccionadas ${ }^{8}$ para el análisis de digamos son: subacto, acto e intervención; inicial, medial y final ${ }^{9}$.

8 Por cuestiones de limitación de espacio, no se explicarán todas las unidades. Para conocer en detalle el funcionamiento de aquellas que no se emplean en el análisis de la partícula, consultar Grupo Val. Es. Co. (2014).

9 La posición independiente no puede ser aplicada para digamos: esta partícula discursiva no tiene un ámbito tan amplio que le permita actuar sobre todo un diálogo. En ese sentido, siempre conservará en su base semántica la acción de decir algo. El tipo de decir puede variar (más modal, más literal...), pero siempre remitirá a este. 


\subsubsection{Intervención, acto y subacto en el modelo de unidades Val.Es.Co.}

\subsubsection{Acto}

El acto es la "unidad estructural monológica, jerárquicamente inferior a la intervención, de la que es su constituyente inmediato". Se caracteriza por ser "aislable e identificable en un contexto dado" (Grupo Val. Es. Co. 2014: 39), "con contenido proposicional que permite distinguir unos actos de otros" (Pons 2016: 554-555), además de "ser la mínima unidad de acción e intención". La siguiente intervención se compone de tres actos, demarcados con el símbolo \# (7):

(7) 42 B: \# [claro $\downarrow]$ /\# \# sí \#/ \# o sea ¿exposiciones de alumno a alumno? o sea de gente que [está allí=] \#

(Conversación 29, Corpus Val. Es.Co. 2.0)

Los tres actos son aislables e identificables porque cada uno posee una fuerza ilocutiva diferente (aseveración, aseveración y petición informativa, respectivamente) y un contenido semántico-informativo también diferente. Son aislables porque podrían funcionar como intervención por ellos mismos, independientes unos de los otros.

\subsubsection{Subacto}

El constituyente inmediato del acto, el subacto, se define como "segmento informativo identificable, habitualmente mediante marcas semánticas y prosódicas" (Grupo Val. Es. Co. 2014: 55). Los subactos se clasifican en dos grandes tipos: subactos sustantivos (SS), con contenido proposicional o sustancia semántica; y subactos adyacentes (SA), sin sustancia semántica y con contenido procedimental (Grupo Val. Es. Co. 2014: 55). Los subactos sustantivos pueden ser directores (SSD), cuando su contenido informativo es el principal dentro del acto, y subordinados (SSS), cuando dependen de un SSD. Por su parte, los SA pueden ser de tres tipos: subacto adyacente textual (SAT), cuando contribuyen al flujo informativo (conectores, por ejemplo), subacto adyacente modal (SAM), cuando modalizan el contenido sobre el cual tienen ámbito, y subacto adyacente interpersonal (SAI), cuando explicitan relaciones interpersonales entre hablantes (8):

(8) 42 B: \# [ $\{$ SSD claro $\downarrow$ SSD $\} / \# \#\{$ SSD sí SSD $\} \# / \#\{$ SAT o sea SAT $\}$ SSSD ¿exposiciones de alumno] a alumno? SSD $\}$ SAT o sea SAT $\}$ SSS de gente que [está allí=] SSS

(Conversación 29, Corpus Val. Es.Co. 2.0)

Hay tres subactos sustantivos directores (SSD) que contienen la información dominante dentro del acto. El marcador o sea, utilizado en dos ocasiones, funciona como SAT, y explicita los dos movimientos de reformulación del hablante. El último subacto sustantivo subordinado (SSS) depende estructuralmente del SSD que le precede. Esta relación entre SSD y SSS supone el puente entre la segmentación de estructuras discursivas y la segmentación sintáctica tradicional: una segmentación discursiva nunca llegará a un nivel de análisis sintáctico. 


\subsubsection{Intervención}

La intervención se define como "unidad máxima monológica estructural, asociada al cambio de emisor, caracterizada por ser o provocar una reacción, generalmente lingüística" (Grupo Val. Es. Co. 2014: 19). En otras palabras, es toda producción discursiva de un hablante dado y relacionada con las producciones discursivas de otros hablantes. La combinación de dos o más intervenciones es un intercambio (9):

(9) Ii - La cuestión es que no funcionaba la comisión técnica...

Ir -Para mí todo tiene que tener una función positiva. Digamos que había cosas con las que no estaba de acuerdo, pero no quiero comentar nada más sobre esto.

Las intervenciones pueden ser iniciativas (Ii) si generan reacciones, reactivas (Ir) si son resultado de una intervención previa, y reactivo-iniciativas (Ir-i) si son reacción y, a su vez, nueva intervención iniciativa. En el ejemplo, la primera intervención es iniciativa y la segunda reactiva.

\subsubsection{Ficha metodológica Es.Var.Atenuación: funciones seleccionadas}

La segunda herramienta de análisis, combinada con la anterior, es la Ficha de atenuación (Albelda et alii 2014), confeccionada en el seno del grupo de investigación Val.Es.Co. y del proyecto Es.Var.Atenuación. Esta ficha está basada en diferentes trabajos (Albelda y Cestero 2011, Cestero y Albelda 2012; Briz y Albelda 2013) desarrollados en el marco de este y otros proyectos pragmáticos y sociolingüísticos, como PRESEEA (Briz y Albelda 2013: 290).

Cuatro son las funciones básicas de la ficha Es.Var.Atenuación, explicadas en la sección 4: función 0 , sin atenuación; función 1, autoprotección de la imagen; función 2, salvaguarda del yo y del tú; función 3, reparación. De estas, tres se aplicarán al análisis de digamos que aquí nos ocupa: función 0 (§4.1.), función 1 (§4.2.), y función 2 (§4.3.). La función 3 (reparación) se sustituirá por los casos de apoyo formulativo (§4.4.).

\section{ANÁLISIS DE OCURRENCIAS}

El análisis que presentamos parte de las ocurrencias extraídas de los corpus conversacionales Val.Es.Co. (Corpus de conversaciones coloquiales Val.Es.Co. (Briz y grupo Val. Es. Co. 2002), Corpus Val.Es.Co. 2.0. (Cabedo y Pons en línea)). Al ser un trabajo inicial, y para obtener unos resultados homogéneos, solo se han utilizado estos dos corpus. De este modo, se evita la posibilidad de mezclar características de otros corpus que, a pesar de recoger muestras orales, no se ciñen puramente al registro coloquial (entrevistas, lenguaje coloquial en adolescentes, imitación de lo oral, etc.).

El total de ocurrencias de digamos en ambos corpus no es muy elevado: un total de 23 muestras (16 en el corpus Val.Es.Co. del 2002, y 7 en el corpus Val. Es. Co. 2.0, de un total de palabras 180246$)^{10}$ que, por otro lado, han permitido un análisis exhaustivo de cada muestra para comprobar si la aplicación de las variables seleccionadas cubre los problemas descriptivos y de sistematización presentes en la partícula.

10 Cifra aproximada, obtenida de la suma del total de palabras en el corpus Val.Es.Co. (2002) y Val.Es.Co. 2.0. 
Digamos puede considerarse un modificador externo (Albelda et alii 2014: 13), puesto que altera la base semántica o pragmática del elemento o segmento sobre el cual tiene ámbito, según el contexto de uso en que se emplee. El grado de modificación subyacente en digamos permite concebirlo desde algunas de las variables de la ficha: digamos 0 (aproximador), digamos 1 (autoprotección de la imagen), digamos 2 (salvaguarda del tú y del yo) y digamos 3 (apoyo formulativo). Además, cada caso se analizará desde la combinación de su correspondiente unidad y posición dentro del modelo Val.Es.Co.

\subsection{Digamos 0 (aproximador)}

Los casos de digamos aproximador entran en la función 0 de la ficha de análisis: no manifiestan propiedades de atenuación semántico-pragmática, sino un valor de aproximación, asociado normalmente a información de carácter numérico.

(10) L: lo mejor se bebee pues§

G: $\quad$ Sen una noche que nos vamos por ahi $\downarrow$ los viernes por la noche que nos vamos por ahi $\downarrow$ pues hay veces que

L: $\quad$ §cuatro- cuatro una media digamos ¿no?§

G:

$\S$ cincoo-

cuatro o cinco se ve que e- e- es que después nos vamos a Cubalitroo

E: y sigue con cubatas§

(Corpus Val. Es. Co. 2002, L.15.A.2)

Como en el ejemplo (10), en estos contextos digamos introduce cantidades aproximadas en un contexto comunicativo determinado. Los digamos 0 están más cerca de la ejemplificación que de la vaguedad semántica y pragmática, pero ya son susceptibles de categorizarse como partícula discursiva: morfológicamente cumplen las propiedades descritas en $\S 2.2$. (primera persona plural, sin sujeto plural, en tiempo subjuntivo y presente); sintácticamente no presentan integración en la estructura de la intervención en que se emplean, por lo que su supresión no daría como resultado un enunciado agramatical (11):

(11) L: $\S \#\left\{_{\mathrm{SSD}}\right.$ cuatro- cuatro una media $\left.{ }_{\mathrm{SSD}}\right\}\left\{_{\mathrm{SAI}} \dot{i n o} ?_{\mathrm{SAI}}\right\} \#$

Semánticamente, no aportan vaguedad al significado global de la intervención, pero sí a la certeza de la información ofrecida: su ámbito, por tanto, no es el del dictum, sino el del modus. Y en cuanto a su análisis con unidades de segmentación, digamos constituye un subacto adyacente modal (SAM), ubicado en posición final del subacto sustantivo director (SSD) sobre el que tiene ámbito (12):

(12) L: $\S \#\left\{\left\{_{\mathrm{SSD}}\right.\right.$ cuatro- cuatro una media ${ }_{\mathrm{SSD}} \mathrm{SAM}$ digamos $\left.{ }_{\mathrm{SAM}}\right\}\left\{_{\mathrm{SAI}} \dot{\mathrm{n} O}\right.$ ? $\left.{ }_{\mathrm{SAI}}\right\} \#$

Estos casos, sin embargo, presentan cierta complejidad que se ve reflejada en su análisis: digamos 0 conservan el puro valor de dicción del verbo original, puesto que al fin y al cabo no dejan de presentar una información o cantidad a modo de ejemplo en un contexto dado. Este hecho lo sitúa en una frontera borrosa entre el enunciado y la enunciación: del enunciado, porque no deja de ser un verbo que explicita la acción verbal del hablante (vamos a decir o hemos dicho, en este contexto, lo siguiente). De la enunciación, porque 
ya en estos casos sería igualmente sustituible por adverbios como aproximadamente, o modificadores como más o menos, siempre con ámbito sobre un ejemplo aproximado (13):

(13) L: §\#cuatro- cuatro una media digamos / aproximadamente / más o menos / pensemos / imaginemos / pongamos por caso ¿no?\#

En cualquier caso, digamos 0 no implica una protección de la imagen del hablante, una salvaguarda del yo y del tú, o una reparación discursiva.

\subsection{Digamos 1 (autoprotección de la imagen)}

Los casos de digamos en sentidos figurados entran en la función 1 de la ficha: se utilizan cuando los hablantes emiten un contenido de manera imprecisa, mediante el uso de metáforas o sentidos no-literales que le ayuden a expresarse en un contexto comunicativo dado. En estos casos de atenuación, el hablante busca salvaguardar su imagen social y evitar responsabilidades sobre lo dicho, en tanto que sus palabras pueden afectar negativamente a su yo (Albelda et alii 2014: 9) (14):

(14) A: [y el]

M: \# que/ ¿entiendes? //\# \# tú ya me c- síi/ las cosas como son/// porque tú estás con tu madre $\uparrow / \#$ \# mi hijo está conmigo $\uparrow / \#$ \#la que mando soy yo $\uparrow / \#$ \#a ver si me entiendes/\# \#el cabeza de familia $\{\mathrm{SAM}$ digamos $\mathrm{SAM}\} / / /$ \# [pero es el único que/ tiene maás// ¿noo?/\# \# ¿tú no crees?]\#

A:

ell- ellos si quieren ponerlo $\uparrow]$ ellos si quieren ponerlo $\uparrow \S$

M:

[ellos-

$\S$ sí

(Corpus Val. Es. Co. 2002, S.65.A.1)

En (14) se observa un funcionamiento de digamos distinto al del ejemplo (13). Aunque las características morfológicas y sintácticas son las mismas, su valor semántico y pragmático difiere. Semánticamente, digamos 1 sí evoca una vaguedad que distancia el significado del segmento en el que se inserta (el cabeza de familia) de su significado original. Ante el uso de una expresión como cabeza de familia, tradicionalmente relacionada con la figura masculina, asociada a una mujer, la hablante marca su enunciación con digamos. Explicita que aquello que acaba de decir no debe tomarse literalmente: $\mathrm{M}$ es quién manda $\mathrm{y}$, por extensión, es el cabeza de familia. Este tipo de casos presenta un grado de atenuación semántico (Briz 1998). Pragmáticamente, M desea proteger su imagen comunicativa ante una posible falta de comprensión por parte su interlocutor.

En cuanto a su segmentación, digamos está en posición final de un acto que está, a su vez en posición medial de la intervención de $\mathrm{M}$. El ámbito de la partícula es el contenido del acto (y, por tanto, de su fuerza ilocutiva) en que se inserta, tal como se aprecia en el fragmento ya segmentado (15):

(15) M: \# que/ ¿entiendes? //\# \# tú ya me c- síi/ las cosas como son/// porque tú estás con tu madre $\uparrow / \#$ \# mi hijo está conmigo $\uparrow / \#$ \#la que mando soy yo $\uparrow / \#$ \#a ver si me entiendes/\# \#el cabeza de familia $\{\mathrm{SAM}$ digamos $\mathrm{SAM}\} / / /$ \# [pero es el único que/ tiene maás// ¿noo?/\# \# ¿tú no crees?]\# 
Digamos 1 no implica una protección de la imagen del interlocutor, puesto que su imagen comunicativa no está siendo invadida por $\mathrm{M}$.

\subsection{Digamos 2 (salvaguarda del yo y del tú)}

Los casos de digamos asociados a protección de la imagen entran en la función 2 de la ficha: el hablante sí busca preservar, no solo su imagen, sino también la de su(s) interlocutor(es). Estos casos de digamos 2, cuyo grado de atenuación es pragmático (Briz 1998), son propios de la interacción oral. Se sabe previamente que la imagen del otro puede dañarse a raíz de su intervención y, por tanto, en el contexto debe explicitarse que esto va a suceder. Opiniones contrarias a las de otro hablante, o actos de habla como los reproches, son ocurrencias prototípicas en las que se utiliza digamos 2 (16):

(16) 158 B: §por cierto $\downarrow$ que se dan cosas muy raras ¿eh? hoy/ no sé/ a mí todo eso dee logopedia $\uparrow$ sicomotricidad $\uparrow \S$

$159 \mathrm{~A}: \quad$ §que se dan cosas muy raras [dee]

$160 \mathrm{~B}$ : [ibueno!]

161 A: yo ¿sabes lo que pien[so $\uparrow ?]$

$162 \mathrm{~B}$ : $\quad$ [no] es que sean raras/ pero que no es $\rightarrow$ un poco digamos/ un poco romper con lo- con laa-/ con lo tradicional $\downarrow$ ¿no?§

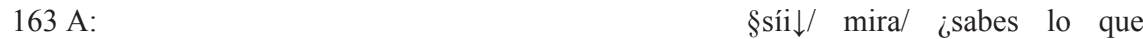
pasa $\uparrow$ ?/ yo es que pienso una cosa $\rightarrow / /$ resulta que a- allíi een-/ en la escuela $\uparrow^{\circ}$ (nos decían $)^{\circ}$ no $\uparrow$ es que el niño $\uparrow /$ no se puedee no sé cuántos $\uparrow$ porque si no un trauma $\uparrow /$ y $\uparrow / /$ yo eso lo encuentro una chorrada $\downarrow$ ¿no? porque/ si no es que siempre estarías haciendo lo que quiere el niño

(Corpus Valesco 2.0, Conversación 1, Intervenciones 158-166)

En (16), el hablante B atenúa en un nivel más pragmático: cuando habla de "romper con lo tradicional" trata de camuflar, con una formulación mitigada y positiva, el hecho de que, en realidad, los métodos de logopedia o sicomotricidad, mencionados anteriormente, le parecen poco adecuados y extraños. De este modo protege su imagen social y, a su vez, evita invadir el terreno de su interlocutor antes de comprobar que, realmente, sí comparten la misma opinión. Es una función preventiva.

Esta caracterización pragmática es la que diferencia digamos 2 de los casos anteriores, a pesar de compartir las mismas propiedades morfológicas, sintácticas e incluso semánticas.

En cuanto a la segmentación con Val.Es.Co., digamos aparece en posición medial de SSS, Estos contextos de atenuación forman parte de lo que se ha definido como digamos 2, orientados a salvaguardar el yo y el tú. Digamos aparece ubicado hacia el inicio del subacto sustantivo director (SSD), aunque su ámbito es todo el acto (un poco digamos un poco romper con lo- con laa-/ con lo tradicional ¿no?), reduciendo su fuerza ilocutiva (aseveración) (17):

(17) $162 \mathrm{~B}: \#\left\{_{\mathrm{SSD}}[\mathrm{no}]\right.$ es que sean raras/ $\left.{ }_{\mathrm{SSD}}\right\} \# \#\left\{_{\mathrm{SAM}}\right.$ pero que no es $\left.\rightarrow{ }_{\mathrm{SAM}}\right\}\left\{_{\mathrm{SSD}}\right.$ un poco $\left\{_{\mathrm{SAM}}\right.$ digamos/ $\left.{ }_{\mathrm{SAM}}\right\}$ un poco romper con lo- con laa-/ con lo tradicional $\left.\downarrow{ }_{\mathrm{SSD}}\right\}\left\{_{\mathrm{SAI}}\right.$ ¿no? $\left.{ }_{\mathrm{SAI}}\right\} \S \#$

$\mathrm{Al}$ introducir una opinión contraria a la de $\mathrm{A}$, digamos funciona en este contexto como un elemento de prevención, de salvaguarda de los yo y tú discursivos. 


\subsection{Digamos 3 (formulativo)}

Los casos de digamos formulativo son los más ligados al registro coloquial. No atenúan ni introducen valores aproximados, sino que permiten al hablante ganar tiempo en su formulación discursiva cuando no se encuentran las palabras necesarias para expresar con claridad una idea.

Por último, pero no menos importante, se registran ocurrencias de digamos como apoyo formulativo (en línea con los ejemplos expuestos al inicio de este artículo). Este es el uso más oral: digamos 3 no atenúa, ni introduce valores aproximados, sino que permite al hablante ganar tiempo en su formulación discursiva cuando no consigue obtener las palabras necesarias para expresar con claridad sus ideas:

(18) 110 A: \# [\{SSD sí sí sí SSD $\} \#$ \#\{SSD es así SSD $\}] \#$ / \#[\{SSD quee ¿cuánto cobran? SSD $\} \#(())\{$ SSS una mierda] SSS $\} /[\#\{$ SSD hay tres tipos de sueldo $\downarrow$ SSD $\} \# \#\{$ SSD HAY unos que son la es CUELA ESCUELA] ESCUELA que es de nueve aa cinco $\uparrow$ de nueve a DOS $\uparrow$ SSD $\} \# / / \#\{$ SSD luego $\downarrow$ los que hacen guardería que es de ocho a dos $\uparrow$ $\mathrm{SSD}\} \# \#\{\mathrm{SSD}$ y los que hacen $\rightarrow$ / ee $\{\mathrm{SAM}$ digamoss $\mathrm{SAM}\} \mathbf{h m m}$ no sé cómo se llama pero es lo de después de comer SSD $\} / /\{$ SSD y es de-de- de ocho $\uparrow$ a cinco $\downarrow$ SSD $\}\{$ SSS que es lo que* quiere hacer Manel por ejemplo que quiere cobrar más que nadie $\rightarrow \operatorname{SSS}\}$ §\#

(Corpus Valesco 2.0, Conversación 42, Intervenciones 106-114)

Así sucede en el ejemplo (18): entre vacilaciones como ee o hmm, seguidas de afirmaciones como no sé cómo se llama, digamos no puede tener ámbito más allá de la estructura del propio subacto en el que se inserta. Sin embargo, la intención final es el mantenimiento del turno de habla para evitar que se produzcan lugares de transición pertinentes (LTP, Levinson 1989) en los que el interlocutor pueda robar el turno en la conversación. Estos casos remiten a la acción de decir, de anunciar nueva información que llega inmediatamente. Es el grado máximo en la escala gradual propuesta en este estudio. Su etiqueta dentro del modelo de unidades Val.Es.Co. sería la de subacto adyacente modal, ya que se explicita el proceso de elaboración del discurso por parte del propio hablante.

Puede observarse que, según el tipo de función atenuadora que cubra digamos, la posición ocupada y el tipo de unidad sobre la que tiene ámbito, su caracterización presenta diferencias. La siguiente rejilla de análisis cruza las variables unidad, posición y función: puede verse como un mismo de unidad, digamos, como SAM en todos los casos, responde a una función distinta no solo gracias a la posición que ocupa, sino también a la unidad discursiva a la que se adscribe. En esta tabla aparecen sin sombrear aquellas unidades y posiciones utilizadas en el análisis de digamos, mientras las no utilizadas aparecen sombreadas (Tabla 2).

\begin{tabular}{|c|c|c|c|c|c|c|}
\hline Digamos & & $\mathrm{S}$ & $\mathrm{A}$ & & & $\mathrm{D}$ \\
\hline & SSS & SSD & & Ir & Ii & \\
\hline I & & & (12): función 2 & & & \\
\hline $\mathrm{M}$ & & (13): función 3 & & & & \\
\hline $\mathrm{F}$ & & (10): función 0 & (11): función 1 & & & \\
\hline Ind & & & & & & \\
\hline
\end{tabular}

Tabla 2. Cruce de funciones de digamos y unidades Val.Es.Co. 
Cuando la función de digamos no implica atenuación (función 0) o solo sirve de apoyo formulativo (función 3), el ámbito del SAM digamos es solo el subacto sustantivo director (SSD). Sin embargo, cuando la función se asocia a la autoprotección de la imagen del hablante (función 1) o a salvaguardar la imagen de hablante e interlocutor (función 2), el ámbito del SAM es el acto y su correspondiente fuerza ilocutiva. Por tanto, la segmentación de los contextos de uso con las unidades y subunidades del modelo Val.Es.Co. ha facilitado la descripción de una partícula que, a pesar de mostrar una instrucción asociada a la vaguedad discursiva, funciona en distintos niveles comunicativos.

\section{CONCLUSIONES}

Tras aplicar la propuesta de análisis Val.Es.Co.-Es. Por. Atenuación observamos que:

- La extensión de la descripción para cada contexto queda más acotada, pero no por ello más limitada. En lugar de un tratamiento abierto, sin un patrón de base, cada caso pasa por delimitar una posición en relación con una unidad concreta. La combinación posición + unidad equivale a cuatro tipos funcionales $(0,1,2, y 3)$ que cubren el espectro de valores de la partícula discursiva digamos. Esto evita un análisis basado en detectar los límites del enunciado y la enunciación, desde una perspectiva abstracta, o cerrándolo a un solo tipo de mitigación, sin matices contextuales y estructurales. Por tanto, existen grados de atenuación con una misma partícula.

- Se observa que unidades como el subacto remiten a valores de digamos de corte más semántico (función de aproximación y función formulativa). Cuando la unidad es el acto, se evocan valores de digamos más pragmáticos (función autoprotección y función preventiva).

- Por el momento, sin embargo, no se han hallado casos de digamos con valor reparador: puede pensarse que se deba a su valor introductor, que anuncia algo que va a decirse. Un significado que, al fin y al cabo, se ha mantenido en la base semántica de la partícula (principio de estratificación, Hopper 1996).

- Por último, queremos resaltar que, si bien el total de ocurrencias en los dos corpus conversacionales es reducido (23 casos, 16 de ellos digamos 1), este trabajo supone un punto de partida para aplicar y replicar este patrón de análisis en trabajos más ampliados: con otras partículas discursivas (tratando de cubrir el paradigma de partículas basadas en verba dicendi) y con otros corpus orales del español (PRESEEA, COLAM, CREA oral, ESLORA2, entre otros).

\section{Referencias bibliográficas}

Albelda, M. y A. Briz (2010). "Cortesía y atenuantes verbales en las dos orillas a través de muestras orales". En Aleza, M. y J. M. Enguita (eds.). La lengua española en América: normas y usos actuales. Valencia: Universitat de València, pp. 237-260.

Albelda, M. y A.M. Cestero (2011). "De nuevo, sobre los procedimientos de atenuación lingüística", Español Actual, 96, pp. 121-155.

Albelda, M., Briz, A., Cestero, A. M., Kotwica, D. y C. Villalba (2014). "Ficha metodológica para el análisis pragmático de la atenuación en corpus discursivos del español. ES.POR.ATENUACIÓN", Oralia, 17, pp. 7-62. 
Blum-Kulka, S. y E. Olshtain (1984). "Requests and apologies: A cross-cultural study of speech act realization patterns (CCSARP)", Applied Linguistics, 5, pp. 196-213.

Bravo, D. y A. Briz (coords.) (2004). Pragmática sociocultural: estudios sobre el discurso de cortesía en español. Barcelona: Ariel.

Briz, A. (coord.) (1995). La conversación coloquial (Materiales para su estudio). Anejo XVI de la Revista Cuadernos de Filología. Valencia: Universitat de València.

Briz, A. (1998): El español coloquial en la conversación. Barcelona: Ariel.

Briz, A. y Grupo Val.Es.Co. (2002). Corpus de conversaciones coloquiales. Anejo de la revista Oralia. Madrid: Arco Libros.

Briz, A. y Grupo Val. Es. Co. (2003). "Un sistema de unidades para el estudio del lenguaje coloquial”, Oralia, 6, pp.7-61.

Briz, A., Pons, S. y J. Portolés (dirs.) (en línea). Diccionario de partículas discursivas del español (DPDE): www.dpde.es (15-6-2017).

Briz, A. y M. Albelda (2013). "Una propuesta teórica y metodológica para el análisis de la atenuación lingüística en español y portugués. La base de un proyecto en común ES.POR.ATENUACIÓN”, Onomazéin, 28, pp. 288-319.

Brown, P. y S. Levinson (1987). Politeness. Some Universals in Language Use. Cambridge: Cambridge University Press.

Cabedo, A. y S. Pons (eds.) (2013). Corpus Val.Es.Co. 2.0: http://www.valesco.es/?q=es/corpus (1504-2016).

Caffi, C. (2007). Mitigation. Oxford: Elsevier.

Cestero, A, y M. Albelda (2012). "La atenuación lingüística como fenómeno variable”, Oralia, 15, pp. 77-124.

Culpeper, J. (2011). Impoliteness: Using Language to Cause Offence. Cambridge: Cambridge University Press.

Fernández Bernárdez, C. (2002). Expresiones metalingüísticas con el verbo decir. A Coruña, Universidade da Coruña.

Fernández Bernárdez, C. (en línea). "Digamos”. En Briz, A., Pons, S. y J. Portolés (dirs.). Diccionario de Partículas Discursivas del Español (DPDE): www.dpde.es (15-6-2017).

Fuentes, C. (2010). Diccionario de conectores y operadores del español. Madrid: Arco/Libros.

González Ruiz, R. (2006). "Enunciados performativos y verbos performativos: acerca de la performatividad como propiedad gradual (con especial referencia al español)", Hesperia. Anuario de Filología Hispánica, IX, pp.107-126.

Grande Alija, F.J. (2010). "Usos metadiscursivos de las formas exhortativas no digamos, digamos y que digamos", Onomázein, 21, pp. 97-131.

Grupo Val.Es.Co. (2014). "Las unidades del discurso oral. La propuesta Val. Es. Co. de segmentación de la conversación (coloquial)", Estudios de Lingüistica del Español, 35, pp. 13-73.

Haverkate, H. (1994). La cortesía verbal: estudio pragmalingüístico. Madrid: Gredos.

Hopper, P. (1996). "Some recent trends in grammaticalization", Annual Review of Anthropology, 25, pp. 217-236.

Morel, M.A. y A. Rialland (1992). "Emboîtement, autonomies, ruptures dans l'intonation française", Travaux Linguistiques du CERLICO, 5.

Narbona, A. (1989). Las subordinadas adverbiales impropias en español. Málaga: Ágora.

Pons, S. (2016). "Cómo dividir una conversación en actos y subactos". En Bañón Hernández, A. M., Espejo Muriel, M. M., Herrero Muñoz-Cobo, B., López Cruces, J. L. (coords.). Oralidad y análisis del discurso: homenaje a Luis Cortés. Almería: Editorial Universidad de Almería, pp. 545-566.

Real Academia Española (RAE). Corpus de referencia del español actual (CREA): www.rae.es (156-2017).

Roulet, E. (1991). "Vers une approche modulaire de l'analyse du discours", Cahiers de Linguistique Française, 12, pp. 53-81. 
Roulet, E., Auchlin, A., Moeschler, J. y C. Rubattel (1985). L'articulation du discours en français contemporain. Bern: Peter Lang.

Sacks, H., Schegloff, E. A., y G. Jefferson (1974). "A Simplest Systematics for the Organization of Turn-Taking for Conversation”, Language, 50, 4/1, pp. 696-635.

Santos Río, L. (2003). Diccionario de partículas. Salamanca: Luso-Española de ediciones.

Schneider, S. (2007). Reduced parenthetical clauses as mitigators. A corpus study of spoken French, Italian and Spanish. Amsterdam/Philadelphia: John Benjamins.

Sinclair, J. y R.M. Coulthard (1975). Toward an Analysis of Discourse. Oxford: Oxford University Press.

Van Dijk, T.A. (1979). "Recalling and summarizing complex discourse". En Burghardt, W. y Holker, K. (eds.). Textverarbeitung/ Text Processing. Berlin/New York: de Gruyter, pp. 49-118.

Zhang, Q. (1998). "Fuzziness - vagueness - generality - ambiguity", Journal of Pragmatics, 29, pp. 13-31. 